\title{
La musicalité magique du rêve colonial à travers Rouge Brésil de Jean Christophe Rufin
}

\section{Par Fathéya AL Fararguy}

Professeur-adjoint

Faculté de pédagogie-Université de Tanta Chercheur associé - centre de recherches sur Zola et le naturalisme de l'Institut des Textes et Manuscrits modernes, UMR 8132 du CNRS-France 
Rouge Brésil écrit par Jean-Christophe Rufin a reçule prix Goncourt en 2001. Il s'agit d'un roman qui a pour cadre les colonies françaises, "Roman colonial » ou de préférence « roman de colonies » ${ }^{(1)}$ comme le définit NobertDodille. A savoir, la colonisationfrançaise en Amérique du Sud : question d'une brulante actualité en France! Quels sentiments, cette partie de l'histoire française, dont les conséquences (les problèmes d'intégration et de sentiment d'appartenance aux valeurs de la République française) sont visibles dans l'actualité récente, peutelle évoquer? Le premier temps de la colonisation française a eu un rôle primordial dans l'orientation de l'œuvre de Rufin.

Lauréat de plusieurs prix pour des essais consacrés aux questions internationales; Rufin est un écrivain académicien, historien et médecin engagé dans l'action humanitaire, exambassadeur de France au Sénégal. Ce plus jeune membre de l'Académie française définit lui-même son roman : «Dans cette histoire marquée par la politique, l'aventure, la théologie, peuplée de guerriers, de fanatiques, de trafiquants, je désespérai de découvrir jamais le frissonnement d'un affect et je la gardai longtemps par-devers moi ${ }^{(2)}$.

1 DODILLE Nobert, Introduction aux discours coloniaux, Paris, Presses de l'Université Paris-Sorbonne, 2011, p.7.

2 RUFIN Jean-Christophe, Rouge Brésil, Paris, Gallimard, 2001, p.601. Dorénavant, nous utiliserons l'abréviation (R.B.) pour Rouge Brésil. Nous voulons remercier Monsieur J.-Ch.Rufin de m'avoir envoyé le roman de la présente étude et d'autres ouvrages. 
Puisque pour Rufin, le romanest « un espace d'évasion ${ }^{(1)}$, l'hypothèse structurante inaugurant cette recherche part des questions suivantes :le rêve colonial ne se caractériserait-t-il pas par une sorte de musicalité et de magie dans ses justifications camouflées ? L'écriture romanesque ressemble à la composition musicale ; les deux sont des créations, l'orchestre peut figurer les personnages du roman qui donne une représentation dans un temps délimité, ils peuvent se déplacer dans plusieurs lieux, ils jouent sur les sentiments; et le public des spectateurs représente le public des lecteurs.

Est-ce que le papier à musique de la colonisation diffèrerait de celui de la musique ordinaire par son impact sur les sentiments et les esprits des auditeurs ?Et que se passerait-il si ce papier à musique jouait sur la corde des religions ou sur la gloire de la patrie ou encore sur la grandeur du roi ? Quels sentiments cette musique peut-elle évoquer ?Comment l'orchestre constitué par les divers musiciens parvient-t-il à parvenir à une sorte d'harmonie ?Comment le chef d'orchestre Villegagnon a-t- il organisé la fondation de la Nouvelle France?

1 Id., Un léopard sur le garrot: chronique d'un médecin nomade, Paris, Gallimard, 2008, p.601. «Le seul pont qui relie les lecteurs au monde intellectuel et dont on puisse dire qu'il joue incontestablement un rôle d'entrainement dans les phénomènes de ventes est constitué par les prix littéraires », p.289. 
A travers Rouge Brésil, comment des relations s'établissentelles entre les colonialistes, l'embarquement forcé, le monde développé, la «colonisation dynamique», « modernisatrice » et les sociétés exotiques, les «territoires endormis », « les esprits arriérés ${ }^{(1)}$ ?Pourquoi Rouge Brésil publié au XXIe siècle a-t-il pour toile de fond le XVIe siècle ?Ou plus largement, pourquoi un roman « colonial » ou de « colonie » au XXIe siècle ? Enfin, l'intrigue du roman colonial est-elle différente de celledes autres types du roman?

Le plan de la présente étude se base sur les éléments suivants: poésie et rêverie qui découlent des intrigues dans un cadre colonial, l'intérêt de la réflexion historique et en troisième centre d'intérêt, le point de vue sociologique, le côtoiement de civilisations différentes.

\section{I- Le roman colonial et la musicalité magiques de ses intrigues variées}

Vers la fin de son livre Un léopard sur le garrot : chronique d'un médecin nomade, Rufin, médecin /romancier considère la rupture de ses habitudes au moment de la maladie de ses patients comme une aventure. Cette aventure deviendra pour le romancier la source de son intrigue. C'est ainsi que l'intrigue de Rouge Brésil tire son originalité du premier métier du romancier.

1 LÉVI-STRAUSSEClaude,De près et de Loin, Paris, Odile Jacob, 1990,p. 30. 
Rufinpense que le médecin ressemble à un chasseur. C'est un bon observateur qui examine son patient et « épie sa proie-le malade-, note ses habitudes, ses goûts, ses craintes et ses désirs ».Le romancier « aussi doit avoir vu pour donner à voir ». Grâce à cette idée, sa création se caractérise par une sorte d'originalité : «Il rend ses personnages vivants en les restituant non seulement dans leur apparence, mais dans leurs déplacements, leurs musiques, leurs appétits ${ }^{(1)}$.

Dans Rouge Brésil, la musicalité coloniale naît d'intrigues variées qui ressemblent aux rythmes diverses des chansons patriotiques, lyriques, religieuses, de danse indienne( ${ }^{(2)}$. Ces intrigues n'accorderaient-elles pas au papier à musique de la colonisation un mythe différent de celui de la musique ordinaire?

Le roman étudié semble contenir deux papiers à musique, l'un créé et joué par les responsables et les dirigeants réunis dans un huis clos et l'autre joué au sein du peuple occupé.D'où émerge le premier indice de la magie de cette musicalité. La voici l'héroïne du roman en témoigne en s'installant avec les indiens :« Je suis d'abord parti dans cette direction, m'attendant à découvrir

1 RUFIN Jean-Christophe, Un léopard sur le garrot : chronique d'un médecin nomade, op.cit., p.289.

2 GHIO Bettina, «Musique \& littérature : quels rapports, quels statuts dans la création ? », Acta fabula, vol. 16, n ${ }^{\circ}$, « Musique ! On lit », Mai-juin-juillet 2015, URL : http://www.fabula.org/acta/document9382.php, page consultée le 26 février 2017. 
une sorte de huis clos de notre société face à elle-même, dans le vide de ces terres nouvelles» dit Colombe(R.B., p. 598).

C'est ainsi que les parties du roman composent la portée de la notation musicale de la colonisation rêvée : le cannibalisme, la contradiction de la fondation de Guanabara dans l'union du corps et de l'âme, puis "sienne"pour indiquer la possession. Le roman se termine par un épilogue intitulé : «A propos de »qui présente le personnage historique de Villegagnon, maestro de cet orchestre.

La première intrigue est tissée autour d'une belle entrée du narrateur recherchant un certain Dom Gonzague dans un monastère qui accueille des orphelins pour les accompagner en Amérique. Il est à la recherche d'enfants doués pour les langues. Avec l'aide d'une conseillère, il choisit deux adolescents orphelins: Just et Colombe qui savent le latin, l'italien et un peu d'espagnol. Mais, confrontés à la rudesse de Belloy, le dernier valet du château et au sein d'un domaine sans maitre, ils trouvent le courage de s'enfuir pour retrouver leur père. Ensuite, ils passentquatre ans à Clamorgan, terre fabuleuse en Italie.

Hélas, ils découvrent que leur père est mort et que la conseillère leur a menti.

Les prénoms des deux adolescents esquissent bien l'intrigue: Colombe est un mot anglais qui désigne un oiseau, 
symbole de paix. C'est aussi une allusion au prénom de Christophe Colomb, découvreur de l'ancienne Amérique. Quant au prénom de Just, c'est une connotation à la justice. Ce choix de prénoms est accentué par leurs attitudes déclarées envers la religion : «À force de voir en Italie les amies de France brandir des croix pour affronter des soldats du pape et tout ce monde prétend venir du même Dieu, Just et Colombe avaient jugé prudent de ne pas trop s'avancer sur cette matière religieuse» (R.B., p. 57).

C'est autour de Colombe que se forme la deuxième intrigue. Une intrigue dont le dénouement est plus long et plus compliqué. Dès la préparation de l'expédition, le départ, la vie à bord, elle se déguise en garçon et prend le nom de Colin. Colombe est présentée comme la sœur de Just, mais vers la fin du roman, le lecteur découvre qu'elle ne l'est pas et qu'il l'épouse.

A leur arrivée, elle décide de quitter Just et de vivre avec les indiens : elle s'était juré de s'appeler désormais EEil-Soleil. L'espoir de n'être fille de personne ne lui rendait pas impossible le rêve d'être née parmi ce peuple qu'elle aimait »(R.B., p. 514). Le surnom d'Eil-soleil est inspiré de la rencontre du romancier d'une fille dans un autobus qui avait «un regard bizarre», «singulier» aux "cils d'un si clair qu'ils paraissaient blancs », « au contraire du ciel, ses yeux figuraient un astre qui enfermait un cercle d'azur $\gg{ }^{(1)}$.

1 RUFIN Jean-Christophe, Un léopard sur le garrot: chronique d'un médecin nomade,op.cit., p.303. 
Avec les indiens, Colombe a pour projet d'apprendre le maniement du grand arc, l'art de tailler les flèches, de traquer le gibier, de partager la vie des guerriers. Elle reste proche des indiens et garde l'esprit de Pay-Lo qui voyait en elle « le regard du grand oiseau sacré qui retient l'âme des morts »(R.B., p.551).

Quant à la troisième intrigue, elle se noue autour de l'aventure et du voyage. Le nouveau monde en est le cadre dès le début, mais on ne sait pas vraiment dans quel pays ils se rendent. Il s'agit de la découverte d'une terre nouvelle seulement arpentée parquelques gentilshommes, aventuriers des mers, ou par des expéditions de marchands. Les deux protagonistes pensent qu'il s'agit de la Turquie d'où ils vont s'enrichir et acquérir leurs droits en revenant à Rouen. Cette intrigue peut captiver l'attention du lecteur dès l'incipit.

La scène du voyage jette un éclairage sur la disparition du monde du passé et dévoile lentement un nouveau monde sur une galère de Méditerranée. Cette scène de départ est décrite et transmise par les yeux des deux adolescents qui partent avec un grand rêve. Elle dépeint aussi la construction de maisons blanches, fraîchement plâtrées, aux portes neuves, maisons d'une France en état d'édification.

D'ailleurs, la découverte de ce nouveau monde est également présentée par une sorte de gradation : la scène des voyageurs qui 
embarquent, une description de trois galères désignées comme«Filles des petits horizons» qui baigne dans «les bleus» : «bleu- blanc du ciel, bleu-vert de l'horizon, bleu-violet de la mer et bleu- gris de l'écume »(R.B., p. 105). Cette image créant une sorte d'enchaînement entre ces filles et l'univers : «Des familles de nuages échevelés, grands et petits mêlés, traversaient l'horizon en courant ». Ce mouvement accompagné d'une sorte de prolifération s'élargit de la mer à l'océan.

Après cette présentation globale, l'auteur décrit en détails chaque galère : la Grande-Roberge pour Villegagnon, sa cour de chevaliers et de savants. La Rosée, plus petit que les deux autres, un bâtiment de commerce avec quelques canons, chargé du matériel et des bêtes. Just et Colombe sont à bord de la Roséele mieux loti des trois navires.

Par cette présentation concrète, Rufin identifie le caractère des voyageurs de l'expédition: «Ils portaient en petit et immobile toutes les violences de cet espace infini dont il s'agissait pourtant d'atteindre les limites »(R.B., p. 61).Le navire comparé à : « un coureur épuisé, qui aspirait les risées » permet d'assimiler la colonisation à «un animal de bât qui reprend de mauvaise grâce son travail, il dérouilla tous ses membres engourdis »(R.B., pp. 162, 82). En un mot, cette exploration n'est que l'espoir d'un omnivore à qui « on refuse la nourriture qu'il 
attend et qui se contentera d'une autre, pourvu qu'elle l'aide à survivre »(R.B., p. 321).Ce rêve fabliau se mêle à la peur d'un monde inconnu.

Le romancier anticipe son intrigue et donne à son roman un rythme d'inquiétant inspirée de la nature de " ce lieu désolé», «accablé de soleil et de chaleur», «montrait assez que la vie des hommes n'y était pas la bienvenue». Il annonce sa vision pessimiste :«L'effort, l'énergie, la sombre volonté que mettent dans l'âme les froidures n'avaient pas leur place dans cette étuve propre à faire croitre une vermine de serpents, d'instincts velus et d'oiseaux peints comme sur soie »explique-t-il (R.B., p.217, 8).

En outre, ce nouveau monde est défini par la comparaison faite entre l'expédition en Orient « Nous y sommes comme chez nous " et une Amérique où le romancier s'est rendu quatre fois : " J'ai tout connu: les fièvres, les cannibales auxquels j'ai finalement échappé par miracle et maintenant ces chiens de Portugais qui nous coupent les mains et les pieds quand ils saisissent nos navires à l'abordage »( R.B., p.16).Rappelons à cet égard,ce qu'Edward Saïd a mentionné dans son livre l'Orientalisme. Pour lui, l'orient « le rival culturel » de l'Europe (l'Occident) est «une partie intégrante de la civilisation et de la culture matérielles de l'Europe ${ }^{(1)}$.

1 SAID Edward W, L'Orientalisme: L'Orient crée par l'occident, Traduit de 1'Américain par Catherine Malamoud, Paris, Edition du Seuil, 2005, p.14. 
C'est ainsi que le cadre spatial limité, au début, à des lieux fermés et petits pour ensuite s'élargir dans une transposition de l'Europe à un autre continent inconnu :la Cathédrale de Rouen, l'auberge, le Monastère, le Havre-de-Grâce, les galères, l'Outremer et l'Amérique, le Brésil, la terre ferme, le domaine de PayLo, la baie de Guanabara (Rio de Janeiro aujourd'hui).

\section{II- Le roman colonial et les obstacles à la fondation de la Nouvelle France}

Pourquoi un roman « colonial » ou de « colonie » au XXIe siècle ayant pour toile de fond le XVIe siècle?

L'époque présentée dans le roman figure le premier temps de la colonisation française ${ }^{(1)}$; « un épisode peu connu de (notre) histoire» ${ }^{(2)}$ déclare Véronique Chaupé. La «France Antarctique » est un terme qui vient ducosmographe, l'abbé Thevet qui avait proposé " équinoxiale " (R.B., p. 347).Le rêve d'une plus grande France et de la présence de la métropole dans

1 BANCEL Nicolas, BLANCHARD Pascal, et VERGÈS Françoise, $L a$ colonisation française, Paris, Milan, 2007, p.5. "C'est en 1895 que, pour la première fois, le terme "colonisation" est utilisé dans la langue française, mais aucun dictionnaire ne le mentionne avant le Larousse de 1931 qui le définit comme un terme "sous lequel les socialistes désignent, en la condamnant, l'expansion coloniale qu'ils considèrent comme une forme d'impérialisme issu du mécanisme capitaliste"», «Trois étapes distinctes marquent la colonisation française: des origines à la Révolution française, une période charnière jusqu'au début de la IIIe république, et une période contemporaine (1870-1962) qui se prolonge sous forme de traces et d'héritages aujourd'hui », p.7. La société d'histoire des colonies françaises est fondée le 6 juin 1912.

2 CHAUPÉ Véronique, http://www.lemonde.fr/culture/article/2014/06/05/rougebresil_4429454_3246.html, page consultée lel5juin 2016. 
ses colonies continue d'intéresser beaucoup de citoyens.D'autre part, une rivalité coloniale éclate aussi entre la nouvelle France et la nouvelle Angleterre, ce qui représente l'esprit de l'époque ${ }^{(1)}$.

Rufin a eu l'idée de ce livre quand il vivait au Brésil au début des années 2000, un an plus tard il le publiera. Il déclare : « Le Brésil me donne l'occasion de goûter pour la première foisune oisiveté totale. (...) Il me semblait renaître. Un siècle d'or commençait pour moi, chez Périclès ${ }^{(2)}$. Sa visite au musée du centre-ville appelé Paço Real enrichit encore son écriture pour l'époque coloniale portugaise.

Par ailleurs, afin de rédiger ce roman, Rufin a consulté plusieurs textes contemporains : des mémoires de protagonistes, des témoignages de voyages, des cosmographes, des anthropophages, des études anthropologiques, des historiques et des libellés. Il a suivi les traces des aventuriers et des premiers voyageurs comme l'historien français Franck Lestringant spécialiste du XVIe siècle, et du protestant Jean Léry auteur d'Un voyage fait en la terre de Brésil et à votre époque(R.B., p. 599).

1 Voir HAVARD Gilles et VIDAL Cécile, Histoire de l'Amérique française, op.cit., p. 26. «Les géographes canadiens ont cependant forgé dans les années 1980 les concepts de Franco-Amérique ou Amérique française dont se servent de plus en plus les historiens attachés à l'étude combinée de l'ensemble des colonies française d'Amérique, Antilles comprises».

2 RUFIN Jean-Christophe, Un léopard sur le garrot: chronique d'un médecin nomade,op.cit., pp.254, 255. 
Cependant, à cause de l'immensité de ce travail, il a souffert de «frustration et de paralysie». Une frustration due à deux raisons : " aucune de ces approches ne correspondait à la représentation imaginaire qu'(il) s'était faite de ces événements», " aucune ne comblait l'envie qu'(il avait) de raconter cette histoire à (sa) manière, en résonance avec (sa) propre vie, (ses) idées, (ses) rêves et surtout en tissant les liens nécessaires avec l'époque présente » (R.B., p. 601).Comme beaucoup de romanciers, citons par exemple Roger Martin du Gard qui était aussi passé par des phases de paralysie parce que sa vision des révolutionnaires (en particulier le personnage de Ménestrel qui représente un révolutionnaire de type bolchevik et qui ne pouvait donc être pleinement pacifiste) ne correspondait pas à la réalité historique. Il a dû réécrire de nombreux passages après s'être documenté notamment auprès de son ami Rohrer.

A travers son personnage de Villegagnon, maestro de l'orchestre colonial, le rêve et les étapes de la fondation de la Nouvelle France sont exprimés. Le nom de ce vice-amiral breton est significatif ; il évoque la ville et le verbe "gagner" conjugué au pluriel. Il dresse son plan de fondation de la Nouvelle France en commençant par la construction d'un fort, puis d'une capitale appelée Fort-Cologny, de Genèbre. Son discours est dominé par l'enthousiasme : «Quand nous serons maîtres de tout le pays », « 
me laisserez - vous seul à faire ce vœu », «Allons, tous ensemble; vive Fort-Coligny », « Vive Villegagnon! Vive l'amiral ! A nous le Brésil »(R.B., p. 64), «A la gloire de Notre-Seigneur », « embarquons-nous, mes frères! La France des Amériques nous attend », «Vive la Chrétienté, vive le roi !»(R.B., p. 253).

Voilà comment il rédige une partition à jouer sur les cordes de la religion, de la patrie et du roi.

Dans leur livre Histoire de l'Amérique française, Gilles Havard et Cécile Vidal posent aussi de cruciales questions sur la réception de l'histoire coloniale : «Le désamour pour l'histoire coloniale tiendrait-il aussi, insidieusement, à l'échec ultime de la colonisation française en Amérique du Nord, échec dont les symptômes seraient le faible développement démographique et économique des colonies et les défaites militaires finales ?», « Les chercheurs de notre pays auraient-ils peur d'écrire une histoire dans laquelle les Français apparaissent, en définitive comme des "vaincus" ?» ${ }^{(1)}$ Pour Nicolas Bancel et Pascal Blanchard, ce retour sur un passé colonial révèle de véritables enjeux politique et idéologique: le rôle de l'État dans l'inscription du passé colonial et de son héritage dans le paysage culturel et politique semble déterminant dans plusieurs pays : en Grande-Bretagne, en Hollande, en Turquie, aux États-Unis et au

1 HAVARD Gilles et VIDAL Cécile, op.cit., p.15. 
Portugal $^{(1)}$. Cette clarification approfondie de la colonisation ou de la décolonisation devient une exigence sociale pour les nouvelles générations. Face à la colonisation culturelle et à la ségrégation sociale, le sujet de la présente étude devient de plus en plus important.

Pour cette raison, le roman de colonie peut exprimer, en quelque sorte, la mémoire collective.Le roman débute en 1555, un matin de juin, à huit heures, au temps d'HenriII. De 1600 à 1760, une politique d'alliance avec les autochtones permet l'existence de la Nouvelle-France, ce qui nécessite beaucoup de«tact et de savoir-faire». Les Français profitent de l'aide des Indiens pour pouvoir résister à la pression militaire des Anglais $^{(2)}$. Mais au XVIe siècle, les activités françaises en Amérique ont longtemps été jugées insignifiantes pour l'historiographe ${ }^{(3)}$.

Plus tard, d'autres philosophies règneront et renouvelleront l'esprit colonial. Avec le temps, la tendance d'une exploitation économique va s'intensifier grâce à la création de marchés et à une augmentation des échanges : «Certains n'hésitaient pas à reprendre l'argument des théologiens du XVIe siècle : Dieu a mis des ressources sur terre à disposition des hommes. Si ceux qui les

\footnotetext{
1 BANCEL Nicolas, BLANCHARD Pascal et VERGÈS Françoise,op.cit., p. or.

2 Cf.Ibid., pp.254, 256, 258.

3 Ibid., p.32.
} 
possèdent ne les font pas fructifier, d'autres sont fondés àle faire à leur place» ${ }^{(1)}$. Un autre motif de l'engagement colonial sous la forme de la responsabilité de ces sociétés« dynamiques »est de civiliser les autres sociétés « endormies », de «proposer de nouvelles approches de la citoyenneté», de «réclamer que leur histoire soit reconnue et leur avenir débattu en commun» ${ }^{(2)}$.

Afin de renforcer cette nouvelle fondation, Villegagnon, qui envoie des lettres au roi et à Coligny, demande des renforts en soldats, de nouveaux colons et des fonds. Il écrit une lettre à Calvin son ami à la Faculté d'Orléans en lui rappelant leur amitié, en lui parlant de la colonie et en insistant sur les grandeurs et la prospérité de La nouvelle France. Il lui annonce son besoin en pasteurs, en filles à épouser pour la stabilité des pionniers : «Villegagnon, retranché sur son îlot, n'était peut-être pas hostile au métissage, mais il soumettait ses hommes à une discipline de fer et leur interdisait tout rapport sexuel avec les Indiens en dehors du mariage ${ }^{(3)}$.

Just l'adjuvant de Villegagnon apparaît comme un des témoins, croyant à ce rêve, ébloui par le paysage intact de la nature, déprimé et opprimé par l'expédition. Just apprend le maniement des armes et s'initie à l'escrime. Il est le bras droit de

1 PHAN Bernard, Colonisation et décolonisation françaises depuis 1850, Paris, Armand Colin, 1999, p.42.

2 BANCEL Nicolas, BLANCHARD Pascal, et VERGÈS Françoise, op.cit., p.25.

3 HAVARD Gilles et VIDAL Cécile, op.cit., p.47. 
Villegagnon : «il épousait désormais (s)es idées sur la France antarctique, tandis que la grandeur de la chasteté, les beautés du sacrifice ne rencontraient auprès des travailleurs du chantier que sarcasmes et sourde hostilité »(R.B., p. 318). Mais d'où émerge cette hostilité ? La vie des immigrants est réglée comme du papier à musique.

Le personnage antagoniste:Quintincondamné pour sa religion inaugure le premier chapitre,déclare l'action et donne une vision critique et sinistre de ce continent d'« une de ces terres maudites », «de mort»,de «poudre blanche si fine, étendue en longues plages, ne fût de la poussière d'os», des «cous grêles de squelettes», de « sinistres craquements sortaient de ces feuillages raides comme des côtes de pendus», des « bouquets de cranes y étaient suspendus »(R.B., p. 177).Cette vision pessimiste est accentuée par la voix du narrateur qui rend compte du sort des voyageurs: « rejetés au milieu du monde sauvage, moins heureux que des bêtes puisqu'ils avaient leur conscience pour souffrir d'être dépouillés, vulnérables et hors de toute pitié» (R.B., p. 165).

Il est à remarquer que l'orchestre de la colonisation se compose essentiellement de deux catégories de musiciens apparemment réunis mais réellement ennemis : les anciens résidents appelés aussi les premiers colons ou colonisateurs de l'île ou les catholiques et les nouveaux arrivés ou les nouveaux 
huguenots ou les genevois ou le demi-peuple protestant ou les réformés. Ces émigrés, soit en fuite, soit venus de leur plein gré se composent de menuisiers, boulangers, chapeliers et autres cardeurs $^{(*)}$. D'autres personnages s'occupent de l'organisation du travail et de l'orchestration de la coexistence de toutes ces corporations. Le maître Imbert organise l'installation des nouveaux arrivés qui sont dans un état dégradé : «Hâves, les gencives saignantes, la bouche collée de soif»(R.B., p.165).

Dès leur arrivée, les deux protestants du Pont et Richer commencent à taquiner Villegagnon sur ses procédures de l'exploration de l'île. Quand Just explique qu'on établit un gouvernorat pour représenter l'autorité du roi, du Pont montre sa condescendance. C'est ainsi que le rêve de cette nouvelle France oscille entre le ton tragique et le ton enthousiaste de Villegagnon. Les tentatives d'union entre les anciens et les nouveaux arrivés forment les points culminants de l'œuvre romanesque.

Pendant la première fête de l'arrivée, les hommes sont mis à genoux avec une croix à la main etle prêtre bénit la foule. Une scène dominée par le grand enthousiasme des combattants, par les acclamations collectives, avec la répétition du verbe vivre quatre fois et l'emploi du pronom nous. Afin de préserver les

* Ouvrier de l'industrie textile qui s'occupait de démêler les fibres en les peignant à l'aide d'une cadre.http://www.linternaute.com/dictionnaire/fr/definition/, page consultée le 20 décembre 2016. 
valeurs de chasteté et de respect et pour peupler la nouvelle société, des fêtes de noces collectives sont organisées. Ces filles sont assimilées à des poussins que la chaleur a fait éclore.Cette comparaison peut évoquer leur innocence.

D'autre part, le narrateur dénonce la vie rigoureuse, la séparation sociale dominée par l'invasion de la solitude des jeunes filles protestantes célibataires en habits noirs ou gris et leurs portraits physiques : Aucune de ces jeunes filles n'était positivement jolie, si l'on considérait des canons esthétiques rigoureux », « aucune de ces Vénus n'était exempte de défauts. Et cependant leur perfection sautait aux yeux » (R.B., p.352).Le parallélisme entre ces deux phrases renforce les causes de leur affaiblissement résumées en deux mots : «L'inactivité et les privations » (R.B., p.523).

Mais bientôt les conflits entre les protestants, les illuminés, les anabaptistes détruisent le rêve de fondation de la Nouvelle France. Par une sorte de confrontation, de parallélisme, le romancier met les anciens en face des nouveaux. Il justifie leurs sentiments contradictoires de honte et de fierté. Honteux de souffrir, ils sont : «efflanqués », « sales», « traqués », « réduits à l'état pitoyable d'une tribu sauvage ».Ils considèrent leur état comme " une insulte à leurs souffrances qu'ils pourraient longtemps tolérer »(R.B., p.348). Leur sentiment de «mauvaise » 
fierté fait référence à leur rôle car il s'agit de leur : « inculquer les dures réalités de la colonie ». Face à cette faiblesse, les nouveaux arrivés sont désignés comme des «proies encore bien innocents » (R.B., p.324) jouissant de la santé, de la force, de l'hygiène. Mais devant l'état déplorable des anciens, les nouveaux arrivés ont décidé de ne pas se laisser arriver à cet état. Voire, pendant la deuxième cérémonie qui a lieu sur la place, les occupants de l'île protestants et catholiques se haïssent et l'étroitesse de l'espace intensifie ce sentiment comme si les deux camps s'examinaient à la loupe ou au microscope.

Au lieu de soutenir les premiers arrivés, les protestants ont commencé à distribuer des bibles et à propager leurs idées « nouvelles », «bizarres » et « singulières » dans un mutisme haineux dénoncé par le ton ironique du narrateur: « des discussions théologiques qui leur faisaient retrouver les précieuses divisions humaines et l'essence même de la civilisation »(R.B., p.360). Colombe remarque qu'il y a un changement dans la colonie, un état de méditation, de convergence, de différence, de surveillance : «De mauvais coups d'œil étaient échangés d'un groupe à l'autre», « Loin de conduire à la concorde et à l'optimisme, ce regain de spiritualité semblait accroître l'hostilité, l'isolement et l'inquiétude »(R.B., p.363). 
Certaines mesures sont prises pour diminuer la violence entre les deux clans : la direction de la prière matinale et la courte série d'oraisons par Thévet imposées chaque matin devant le gouvernorat de Villegagnon.Face à ce bouleversement,l'accent est mis sur le rôle du Cordelier : «On ne pouvait le blâmer de son indifférence religieuse. Il était à l'image de cette église de France, tout entière tournée vers les intérêts séculiers» (R.B., p.288). L'amiral prononce un discours incitant tous les habitants au travail pour mettre fin aux disputes.

Toutefois, les étapes de la rupture avec les nouveaux arrivés se précisent et l'île est partagée en deux territoires adverses, et un contact devient une sorte de trahison. Pour les anciens résidents, c'est la question de supériorité par le nombre qui fait éclater le conflit : «Les catholiques, trop contents de voir s'affaiblir peu que ce fût le camp adverse, avaient accepté de le pourvoir d'eau et de vivres » (R.B., p.437).Pour l'autre camp, moins nombreux, il trouve son soutien en ses chefs du Pont et Richer, forts de leur spiritualité.

Vient enfin le moment du départ des protestants dont la description est comparée à un cri et une révolte de la nature : des pluies lourdes, des orages, des bagages gonflés, «Les malheureux réfugiés grelottaient de froid et de fièvre, recroquevillés, les genoux dans les bras, pour tenir un semblant de chaleur au-dedans 
d'eux »(R.B., pp.500-1).La scène de la pluie et de l'hiver est décrite avec finesse et accentue le rôle « fécondant des saisons ». Le voilà, cet «écrivain-peintre ${ }^{(1)}$ démontre l'influence de ce moment sur lui dans Un léopard sur le garrot: « Il me fallait un hiver pour enfouir ces idées, retourner mon esprit », un moment comparé à « une terre labourée, étendre la paix de la pluie et du gel sur les herbes folles de mes pensées ». Il attend avec les autres saisons, le produit de cette terre: «Ensuite viendraient de nouvelles pousses et peut-être l'espoir d'une récolte» ${ }^{(2)}$.

Ayant pour tâche d'examinerles protestants avant leur départ, Just vit un conflit intérieur. Comme le héros d'Henry de Monterhlant dans La rose de sable, convaincu de la colonisation, le lieutenant d'Auligny est lui aussi confronté au réel.Just est ébloui par la beauté de la protestante, Aude, native de Lons-leSaunier, qui a perdu ses parents dans des persécutions et a été élevée par un oncle. Elle a demandé à Just de tuer Villegagnon. Désespérée de réaliser son souhait, elle a poignardé Just avant son départ. C'est ainsi qu'au lieu de combattre les indiens, une

1 RENARD Jean-Pierre, «Jean-Christophe Rufin: post-modernité et radicalisation », in Territoire en mouvement Revue de géographie et aménagement [En ligne], 13 | 2012, mis en ligne le 01 janvier 2014, consulté le 21 février 2017. URL : http://tem.revues.org/1647.

2 RUFIN Jean-Christophe, Un léopard sur le garrot : chronique d'un médecin nomade, op.cit., p. 257. 
guerre civile entre protestants et catholiques est déclenchée ${ }^{(1)}$. L'échec de cette union n'est-il pas un autre indice de la musicalité magique qui joue sur les cordes de la religion?

Les protestants, au lieu d'être un soutien matériel pour la fondation de la nouvelle France, sont ainsi partis pour la terre ferme sous la direction de Du Pont et ont été reçus par Martin, ils vont y rester trois mois : «Ils s'y étaient organisé une vie régulière faite de prières, de tours de garde en direction de la plage et de la forêt, pour déjouer une éventuelle attaque de Villegagnon »(R.B., p.521). Ce sont les indiens, d'abord dédaignés, qui les nourrissent.

Rouge Brésil est inauguré par un exergue de Michel de Montaigne. Rappelons aussi qu'au XVIe siècle sont publié par Montaigne, les Essais, qui comporte des passages d'une critique radicale de la colonisation européenne, un nouveau regard sur les indiens; le début d'une anthropologie. Au trente et unième chapitre intitulé Les cannibales, il constate : « Cette découverte d'un pays infini semble être de considération. Je ne sais si je me puis répondre qu'il ne s'en fasse à l'avenir quel qu'autre, tant de personnages plus grands que nous ayant été trompés en celle-ci. J'ai peur que nous ayons les yeux plus grands que le ventre, et

1 http://l-or-des-livres-blog-de-critique-litteraire.over-blog.com/article-la-rosede-sable-de-henry-montherlant-121724611.html, page consultée le 30 octobre 2016. 
plus de curiosité que nous n'avons de capacité ${ }^{(1)}$. Montagne met en valeur le côté humain des indiens vus et jugés comme des berbères, comme des cannibales et des sauvages. Ils sont plus humains que leurs envahisseurs.

Cette vision est mise sur scène à travers la discussion de Colombe avec Aude sur la philosophie de l'action missionnaire : «Vous assimilez la foi à une marchandise. C'est bien ainsi que la conçoivent les papistes qui font commerce de gestes et de prières. Hélas, voyez-vous, la foi n'est pas affaire de gestes mais de grâce» déclare-t-elle (R.B., p.376).

Ces batailles théologiques ne figurent-elles pas une sorte de radicalisme?

Rufin, écrivain engage ${ }^{(2)}$ critique ainsi les guerres de religion et la violence décrite comme «une providence pour les criminels», une «violence [qui]tout à coup devient sainte »(R.B., p.477). Il dénonce la justification de tous les actes de cette violence : «Qu'il tue, qu'il vole, qu'il jouisse : seul Dieu peut lui accorder Sa grâce et le tirer, s'il le veut, de cette luxure »(R.B., p.367).Pour aboutir à la réalisation du rêve de l'exploration du nouveau monde, Rufin identifie le statut de la guerre dans

1 MONTAGNE Michel de, Les Essais, p.131. www.livrefrance.com.

2 http://www.lesideesmenentlemonde.fr/821-jean-christophe-rufin.htm, entretiende Jean Christophe Rufin avec Philippe Lapousterle, Les idées mènent le monde, page consultée le 11 novembre 2016 ; 
laquelle la France s'est engagée : «Insérée dans la rivalité continentale de la France et de l'Empire, cette tentative de colonisation du Brésil est également une répétition générale des guerres de religion »(R.B., p.599). Il trace l'état de la société avec la fin de la guerre de cent ans par des images métaphoriques: «Les greniers étaient gorgés de récoltes », «par tout le pays ruisselaient les étoffes et les vins, les épices et l'ouvrage délicat des artisans »( R.B., p.33).

Quant aux illuminés, la troupe des chevaliers de Malte qui« vivent encore comme au temps des croisades » (R.B., p.74) et aux anabaptistes : «hommes hâves, les cheveux blonds laissés longs », « vêtus de blouses de lin », « ennemis du monde » qui maintenaient «sur leurs lèvres un permanent sourire d'extase, comme s'ils eussent capté dans le silence mélodieuse voix divine qui eût chanté pour eux des cantiques ». En un mot, ilsrêvent de se séparer du monde, «mettre à bas les rois, les Églises, toutes les coutumes »(R.B., p. 109).

Avec tout ce qui précède, il y a aussi la colonisation rivale du Portugal, qui avait la terre du Moripon au sud de l'Amérique, pays qui fait la guerre contre Villegagnon. A l'échec de la première tentative de colonisation française s'ajoutent d'autres difficultés : les forêts impénétrables, les conditions climatiques extrêmes, les maladies, l'existence d'espions, Cadorim Vittorio 
qui a le mot de passe, Ribière espion à la cour à Paris. Par ailleurs, les bandouliers ont pour plan d'isoler et d'abattre. Les immigrants deviennent révoltés et découragés parce que certains profitent des richesses et d'autres ne gagnent rien.

\section{III-Roman de colonisation ou d'accostage des civilisations ?}

Dès l'incipit du roman, l'idée qu'il existe des cannibales, c'est-à-diredes mangeurs d'hommes est évoquée entre deux participants à l'expédition. Cette crainte envers les cannibales semble souligner le but humain de cette expédition : lutter contre le cannibalisme pour sauver les indiens.

Cependant c'est la haine qui domine dans des interrogations adressées à un indien désigné par «Malandrin, pendard». Le matelot trace une image dépréciative dès l'incipit du roman : « Imaginez un instant, monseigneur, ce que peut ressentir un homme qui voit bouillir devant lui l'eau où il va cuire »( R.B., p.13). Le verbe « imaginez » incite le lecteur à réfléchir sur le statut de l'idéologie d'exploration de l'Amérique. Mais, pour dévoiler l'objectif réel, le romancier met en scène la recherche des ressources du Brésil. Depuis trois générations, on y cherche le fameux bois brésilien, l'or, les perroquets, les teintures. De ce fait, la couleur rouge annoncée dès le titre du roman fait référence à plusieurs réalités : le bois rouge dont on tire une 
teinture de même couleur et la couleur rouge du sang pour connoter la violence.

Dans Rouge Brésil, c'est la voix des français qui domine, on n'écoute pas la voix des indiens colonisés : « des populations qui n'étaient pas forcément a priori hostiles à des contacts avec les étrangers ${ }^{(1)}$.

Ainsi, par une sorte de confrontation entre lutte contre les cannibales et recherche de ressources; le lecteur est mis dans la situation d'un enquêteur.

Les anciens et nouveaux Français arrivent à se haïr, alors que les Indiens désignés comme guerriers ne montrent aucune hostilité : «Les guerriers serraient les mains des arrivants, leur donnaient l'accolade, leur faisaient dons de plumes et d'os taillés »(R.B., p.188).

C'est autour de la question du cannibalisme, de la normalité, de l'esclavage et de l'attachement à la nature que se forme le monde indien dans le roman. Le seul personnage indien présenté, vivant à Rouen, racheté par des marins français et libéré par Catherine de Médicis, est désigné par "il". Pas même d'indication sur son prénom. A cette époque, on invite des

1 PHAN Bernard,Colonisation et décolonisation françaises depuis 1850, Paris, Armand Colin, 1999, p.15. 
indiens à la Cour du roi pour leur intégration politique ${ }^{(1)}$. Amoureux de Rouen dès son arrivée, cet indien y erre, dort à l'ombre de la tour Nord. On lui recommande de se couvrir en présence de l'ordinaire des français: «Le malheureux s'était rendu coupable de plusieurs audaces en cette matière, dévoilant en public ses parties les plus intimes avec l'innocente intention d'y apporter de la fraîcheur »(R.B., p.17). Il se marie à une normande qui l'encourage à rester en France. Mais il repense souvent à la nature du Brésil qui « lavait son âme de tout le gris dont le quotidien de Rouen l'avait saturé»(R.B., p.17).

D'un autre côté, le romancier présente Le Freux qui respecte les traditions de plumasserie des Indiens, leurs danses, leurs chants nocturnes, les colliers de vignots et les bracelets de coquillages qui ornent leurs cous : «C'était d'ailleurs cette normalité qui rendait gênante leur complète nudité. Rien dans leur apparence ne permettait de les comparer aux bêtes, que l'on est accoutumé à voir paraître au naturel de leur poils »(R.B., p.185).

Cependant, la vision des indiens s'aiguise d'un deuxième élément: l'esclavage: « nos esclaves » dit Villegagnon. L'esclavage pour Gilbert Buti est incompatible avec le nom même du royaume: «Théoriquement les esclaves sont admis

1 HAVARD Gilles et VIDAL Cécile, op.cit, p.277. 
dans le royaume pour y "apprendre un métier et la religion catholique"» ${ }^{(1)}$.

Pour inclure les indiens dans la société coloniale, on essaie de les « civiliser ${ }^{(2)}$. Néanmoins, les protestants estiment que la rédemption des Indiens était impossible. Pour d'autres, les indiens sont des sauvages «gourmands » qui ont «la tête dure».

Colombe, narrateur-personnage, piquée par la conscience de Rufin, ancien président d'Action contre la famine,incarne une focalisation interne. En côtoyant les indiens et en particulier l'un d'entre eux, la question de l'accostage chez elle, occupe une grande place: «Ce moment éphémère et unique recèle une émotion particulière ; bien qu'elle concerne des sociétés, elle s'apparente à l'élan amoureux qui peut saisir deux êtres lorsqu'ils sont mis en présence pour la première fois »(R.B., p.598).

Le deuxième chapitre est inauguré par la description de l'état de Colombe qui se déplace librement entre l'île et la terre ferme. Elle décide de s'éloigner du rêve de la France antarctique et prévoit la trahison d'Aude dont Just est tombé amoureux.Pour

1 BUTI Gilbert, « Gens de couleur et esclaves en Provence au XVIIIe siècle », in Cahiers des Anneaux de la Mémoire, $\mathrm{n}^{\circ}$ 13, 2010, p.316.« En 1625, le roi Louis XIII accepte de faire une exception à cette règle car, selon ses conseillers, l'esclavage faciliterait la conversion des africains transportés, l'esclavage faciliterait la conversion des Africains aux Antilles; l'accord du Roi est toutefois donné à condition que le principe général de la liberté soit maintenu en France », p.309.

2 HAVARD Gilles et VIDAL Cécile, op.cit., p.281. 
elle, Villegagnon est «un monstre ivre de sa puissance »(R.B., p.465).

Avec les scènes dialoguées, la pluralité des voix, Colombe s'attache aux indiens et vit avec Pay-Lo en paix. Avec eux, elle oublie le monde et adhère à leur culture : «Aimer la forêt, c'était porter sur les efforts de la colonie le regard le plus impitoyable, formuler le jugement négatif le plus radical »(R.B., p.391). Dans Le Grand cœur de Rufin, il montre le rôle de la nature « L'observation me fit comprendre ce qui était à mes yeux un mystère » (R.B., pp.220, 221). Colombe apprend le dialecte des indigènes et elle laisse son influence sur son entourage : « Sa présence d'Européenne, quoiqu'elle la voulût calme et discrète, rompit par des brusqueries l'harmonie indienne. Les femmes l'initièrent à des rudiments de conversation qui lui furent assez vite familiers. Mais combien plus difficile était la grammaire des corps. Tout son instinct de sentir les émotions humaines était dérouté dans ce nouvel univers » (R.B., p.237). C'est une image appréciative qui est transmise par Colombe qui utilise même l'expression de « grammaire des corps » pour décrire l'art de leur vie et de leur langue.

$\mathrm{Au}$ sein de la nature de l'Amérique latine, elle fait une comparaison entre la société européenne et celle du nouveau monde: «Mais nulle part comme en Amérique latine, on ne 
trouve proche, encore vivant, presque visible lorsque l'on contemple les paysages côtiers, la trace de ce premier et dramatique accostage, par lequel une civilisation a pris pied dans une autre »(R.B., p.598).

Elle encourage les indiennes à fuir et à se jeter sur les protestantes pour déchirer leurs vêtements. C'est un acte qui accorde une chance aux indiennes de se libérer de leur soumission.Un acte qui dévoile l'identité véritable d'Aude. Mais pour celle-ci, cet acte a précipité la séparation entre les hommes, a mis le désordre, et Just croit, lui-aussi, que Colombe agit contre l’intérêt général.

Elle se dévêt comme les indiens, et découvre qu'il y a des indiens qui mangent leurs semblables.Etonnée, elle pose la question à Pay-Lo qui lui explique que les indiens s'inspirent de la nature. Ils mangent leurs ennemis pour assimiler leur puissance : « Ils ont appris cela de la forêt où tout se compénètre et se féconde, où ce qui n'est pas dévoré dévore »(R.B., p. 340). Quand Colombe se révolte et pense au changement de ce monde, Pay-Lo lui explique : «nous sommes l'un et l'autre nées dans un monde où ce qui est normal, c'est de détruire son ennemi. Les indiens, eux, se l'incorporent. Ils ont l'admirable qualité de se nourrir de ce qui leur est opposé $»$.Toutefois, ils ont un pacte avec la nature : «Tout est sacré, pour eux, les fleurs, les rochers, 
les eaux qui courent dans la montagne. Une infinité d'esprits habitent et protègent les objets, les paysages et les êtres »(R.B., p.510). Comme Colombe, qui se passionne pour la nature, il critique cette colonisation: «Villegagnon, avec ses outils de guerre, attaquait le bloc opaque de la nature brute avec l'enthousiasme de l'artiste qui se met face au marbre de carrière pour en tirer une pietà »(R.B., p.267).

De la question de l'accostage colonial provient une autre question de relation entre soi et l'Autre : «L'Amérique française apparaît en effet comme un théâtre spectaculaire de la relation de l'Autre », "Cette découverte de l'altérité, réelle ou fantasmée, qu'incarnent par excellence les autochones, invite à une réflexion sur soi-même et sur sa propre société ${ }^{(1)}$. La France tenait un rôle prédominant à tel point que le français était pour les Brésiliens enseigné comme seconde langue ${ }^{(2)}$.

Mais, avec ce rêve de métissage culturel, les Indiens ont connu la privation de leur liberté et du cadre spirituel de leur vie: «en les arrachant à leurs tribus », « en les mettant sur cette île d'où toute vie naturelle avait peu à peu disparu », «En quelque sorte, ils étaient déjà morts et acceptaient ce surcroît d'existence comme une inéluctable damnation »(R.B., p.379). Les indiennes ont subi des harcèlements.

\footnotetext{
1 HAVARD Gilles et VIDAL Cécile, op.cit.,p.21.

2 Cf.LÉVI-STRAUSSEClaude, De près et de Loin, op.cit., p. 30.
} 
Just, lui, a juré de défendre cette terre au nom et en mémoire de son père pour le bien de la France, mais il devient confus : « Sa vie entière suait la lâcheté, l'indécision et l'erreur. Sous ses poses de noblesse et d'élégance, il n'avait jamais pratiqué que les plus grossiers compromis et supporté les mensonges qu'il s'inventait, en faisant mine de les croire » (R.B., p.536). Se sentant coupable, il se remet en question : «De quoi étaient-ils capables, lui et ses semblables, sinon d'édifier des murs, de séparer, de diviser, de contraindre ?» (R.B., p.428). Villegagnon va à Paris pour rencontrer le roi et demande à Just de le remplacer et de prendre le commandement de la colonie. Jusqu'à la fin du roman, Colombe reste convaincu du projet de la fondation de la France antarctique.En tant que responsable, Just fait le bilan entre l'Europe et le Brésil : «Ecoute, Colombe, reprit-il, j'ai bien pesé la situation ces derniers jours. De quelque côté que l'on se tourne, je ne vois que la mort. En Europe, le fanatisme se déchaîne, des factions se déchirent pour un Dieu. Et ici, c'est le monde cannibale, avec ses horreurs » (R.B., p.568).

Dans son article intitulé : «Politiques romanesque du fait colonial », Carpanin Marimoutou constate que les romans des colonies ou les romans qui parlent peu ou prou des colonies montrent : " la violence des rapports de domination fondés sur une conception de l'inégalité des "races" et des cultures. Mais le 
monde colonial fut aussi, de manière non voulue et non pensée peut-être, un espace diversifié de contacts et de métissage, d'échanges de textes, d'imaginaires, de mythes, de conception du monde $»^{(1)}$.

G. Havard et C. Vidale dans L'histoire de l'Amérique française, traitent la nature de la relation, franco-indienne : « l'historiographie est marqué par la thèse du génie colonial : en tant que peuple, les Français auraient eu une aptitude particulière à s'entendre avec les autochtones, et en Amérique du Nord plus qu'ailleurs. Leur approche des Indiens aurait été beaucoup plus conciliante et tolérante que celle de leurs concurrents européens» ${ }^{(2)}$.

Si Just a recours à Colombe, c'est parce qu'elle connaît bien les indiens qui peuvent les soutenir contre la menace portugaise ; elle, à son tour s'interroge : "Sauver quoi, Just? La France antarctique ? Elle prononça ces derniers mots en faisant un effort, comme on use maladroitement d'un outil que l'on vient d'emprunter »(R.B., p.568).

Dans La Pensée métisse, Serge Gruzinskiévoque la question de l'uniformisation née du mélange de l'Ancien Monde et le Nouveau Monde. Le cadre temporel du roman de la présente

1 MARIMOUTON Carpanin, «Politiques romanesques du fait colonial », in Les Cahiers Naturalistes, $\mathrm{n}^{\circ} 88,2014, \mathrm{p} .11$.

2 Ibid., p.251. 
étude évoque ainsi la première vague du métissage culturelle.A la fin du roman, la discussion autour de la colonisation, du métissage culturel est cristallisée en un dernier moment de triomphe pour Just et Colombe : «Après un dernier salut à l'île, ils prirent le galop sur le sable et rejoignirent les Indiens.Leur bonheur désormais appartenait à cette terre, une terre qu'ils défendraient toujours mais ne chercheraient jamais à posséder » (R.B., p.592). L'intégration de Colombe, l'héroïne au sein des tributs indiens montre la domination de la culture indienne sur la culture occidentale incarnée par cette jeune fille. Mais les tentatives de pénétration culturelle aux sein des indiens, n'ont pas laissé leurs empreintes.

Rouge Brésilcomprend un aspect théâtral par les dialogues à voix multiples. Selon B. Valette, le dialogue «sert à la représentation directe de la parole », « apparaît le plus souvent comme un "genre intercalaire" ${ }^{(1)}$. Le roman comprend aussi des discours, des messes comme une formule magique, des scènes de voyage et de l'équipage, une longue lettre adressée à Calvin qui montre les pensées de Villegagnon.

Rappelons à cet égard, la loi du 23 février 2005. G. Havard et C. Vidal rapportent qu'on prescrit d'enseigner la colonisation de manière positive dans les programmes scolaires mais

1 VALETTE Bernard, Le roman: initiation aux méthodes et aux techniques d'analyse littéraire, Paris, Armand Colin, 2011, p. 34. 
reconnaissent : " la loi à laquelle s'est opposée une pétition de plusieurs centaines d'historiens et qui manifestent l'attachement persistant de la République pour "l'œuvre coloniale"») ${ }^{(1)}$. C'est une question d'une brulante actualité en France. Mentionnons à cet égard, la polémique actuelle sur Emmanuel Macron, le candidat à la présidentielle qui a qualifié la colonisation française de « crime contre l'humanité » en Algérie ${ }^{(2)}$.

Dans la conclusion de son article intitulé : Regards sur la littérature coloniale française de l'entre-deux-guerres, DanielHenri Pageaux constate que « les jours de cette littérature (coloniale) sont comptés (...) en raison de la difficile survivance de fragments d'épopée, présentée comme pacificatrice et civilisatrice, face à la réalité d'une colonisation qui infirmait ou invalidait le fondement "moral" du projet "colonial"» ${ }^{(3)}$.

1 HAVARD Gilles et VIDAL Cécile, op.cit., p.16.

2 http://www.liberation.fr/elections-presidentielle-legislatives, page consultée le 15 février 2017.

3 PAGEAUX Daniel-Henri, « Regards sur la littérature coloniale française de l'entre-deux-guerres », in Les Cahiers naturalistes, ${ }^{\circ} 88,2014$, p.58. 


\section{Conclusion}

L'intrigue de ce roman colonial compliquée, camouflée, variée rend une image féconde du rêve colonial. Elle nous a inspiré l'idée de la musicalité magiquedu papier à musique de cette colonisation ; une aventure vers un espace infini, vers un monde inconnu.

Pour ce nouveau monde, plusieurs noms ont été utilisés : «le Nouveau Monde », «La nouvelle France», « Le nouveau royaume du Brésil », «La France des Amériques », « la France antarctique », « prometteuse colonie », (R.B., pp. 44, 118, 250, 347, 539), « l'Amérique française ${ }^{(1)}$.

Rouge Brésil est un roman de colonie fondé sur une rivalité entre la construction continentale et la destruction de la nature, entre l'intégration et la désintégration, entre la soif de la recherche de terres vierges et le devoir de civiliser, entre les races inférieures et la suprématie intellectuelle etla rivalité religieuse.

L'espoir de retrouver le père de Just et de Colombe, le rêve d'un métissage culturel, celui d'une constitution d'une existence unanimiste en rassemblant des groupes, des foules, le rêve d'avoir des enfants pour la fondation de la France antarctique, tout cela existe dans ce roman.

1 HAVARD Gilles et VIDAL Cécile, Histoire de l'Amérique française, op.cit., p. 21 
Cependant, « La sourde hostilité » ronge ce nouveau monde composé de plusieurs communautés qui s'entremêlent : les Français, les Portugais etles Indiens.

En un mot, la disparition de ce rêve nous est présenté par une image très éloquente: " petit serpent de crayon qui se mordait la queue sur le papier $»$.

La colonisation est aussi comparée à : « un coureur épuisé » qui « aspirait les risées » (R.B., p.162) ou à « un animal de bât qui reprend de mauvaise grâce son travail.Et le romancier annonce sa vision pessimiste de « la volonté sombre » de ces conquérants, devant un espoir « omnivore».

Le peu de traces d'indianisation des protagonistes est très remarquable sur le plan romanesque de Rouge Brésil par rapport aux personnages rêveurs de la fondation de la nouvelle France. Colombe se laisse absorber par la nature et se livre à sa joie de vivre. Elle a pu garder sa transparence intérieure comme le dévoile la trame narrative, de la situation initiale à la situation finale. Entre Colombe, indianisée au Brésil, qui perpétue l'influence française, et un indien occidentalisé se forme la vision du métissage du nouveau monde.

Par l'influence du jeu de l'éventail, le roman s'ouvre sur un débat sur le cannibalisme entre un français et un indien et se 
termine sur le même sujet entre Colombe et Pay Lo par l'analyse de la vie des indigènes indiens.

L'Amérique est le produit de couches d'histoires successives et La France actuelle connaît aussi une société hétérogène. Le roman de colonie peut exprimer, en quelque sorte, la mémoire collective. 


\section{BIBLIOGRAPHIE SELECTIVE}

\section{CORPUS DE TRAVAIL}

RUFIN Jean-Christophe, Rouge Brésil, Paris, Gallimard, 2001.

\section{AUTRES OUVRAGES DE JEAN-CHRISTOPHE RUFIN}

-La dictature libérale, Paris, J.-C. Lattès, 1998

-Un léopard sur le garrot : chronique d'un médecin nomade, Paris, Gallimard, 2008.

-Le grand cour, Paris, Gallimard, 2014.

\section{OUVRAGES GÉNÉRAUX}

-BANCEL Nicolas, BLANCHARD Pascal, et VERGÈS Françoise, La colonisation française, Paris, Milan, 2007.

- BAMMOU Lahcen, Université ChouaibDoukkali: Culture et interculturalité dans

Si c'est un homme de Lévi Primo, colloque international, Maroc, 2014. -CUISENIER André, Jules Romains: l'unanimisme et Les hommes de bonne volonté,

Paris, Flammarion, 1969.

- DODILLE Nobert, Introduction aux discours coloniaux, Paris, Presses de l'Université Paris-Sorbonne, 2011

-GAZAGNADOU Didier, L'anthropologue sans Orient ni Occident (Des fausses et

Colloque

Dangereuses notions d'Orient (sharq) et d'Occident (gharb),

international «Langues, Cultures et Médias en Méditerranée : Textes,

Discours, Tics/Tices et Frontières », Maroc, 2014.

- GRUZINISKI Serge, La Pensée métisse, Paris,Fayard, 1999.

-HAVARD Gilles et VIDAL Cécile, Histoire de l'Amérique française, Paris, Edition

du Pacifique, 2004.

-LÉVI-STRAUSSE Claude, De près et de Loin,Paris, Odile Jacob, 1990.

-PHAN Bernard, Colonisation et décolonisation françaises depuis 1850, Paris 
Armand Colin, 1999.

-SAID Edward W, L'Orientalisme : L'orient crée par l'occident, Traduit de l'Américain par Catherine Malamoud, Paris, Edition du Seuil, 2005.

- VALETTE Bernard, Le roman : initiation aux méthodes et aux techniques d'analyse littéraire, Paris, Armand Colin, 2011.

\section{AUTRES OUVRAGES}

- MONTHERLANT Henry de, La rose de sable, Paris, Gallimard, 1968.

\section{ARTICLES}

- BUTI Gilbert, «Gens de couleur et esclaves en Provence au XVIII siècle», in

Cahiersdes Anneaux de la Mémoire, ${ }^{\circ}$ 13, Nantes 2010, p.309.

- GHIO Bettina, «Musique \& littérature : quels rapports, quels statuts dans la

création ? », Acta fabula, vol. 16, $\mathrm{n}^{\circ}$ 5, « Musique ! On lit », Mai-juin-juillet 2015, URL : http://www.fabula.org/acta/document9382.php, page consultée le 26 février 2017.

-RENARD Jean-Pierre, « Jean-Christophe Rufin : post-modernité et radicalisation $»$,

inTerritoire en mouvement Revue de géographie et aménagement [En ligne],

13 | 2012, mis en ligne le 01 janvier 2014, consulté le 21 février 2017.

URL :

http://tem.revues.org/1647

- PAGEAUX Daniel-Henri, «Regards sur la littérature coloniale française de l'entre-

pp. 45-59.

deux-guerres », in Les Cahiers naturalistes, $\mathrm{n}^{\circ} 88,2014$,

\section{SITOLOGIE}

http://search.openedition.org/index.php?op[]=AND\&q[]=+coloniale+litt\%C 3\%A 9rature\&field []$=A l l \& s=$ Questions $+\mathrm{de}+$ communication

- CHAUPÉ Véronique, http://www.lemonde.fr/culture/article/2014/06/05/rougebresil_4429454_3246.html, le 5juin 2014.

- http://www.linternaute.com/dictionnaire/fr/definition/ 
-http://www.liberation.fr/elections-presidentielle-legislatives-

2017/2017/02/15/

-http://www.lexpress.fr/actualite/politique/elections/la-colonisation-crimecontre-l-humanite-macron-sous-le-feu-des-critiques_1879756.html

-http://l-or-des-livres-blog-de-critique-litteraire.over-blog.com/article-larose-de-sable-de-henry-montherlant-121724611.html

http://www.persee.fr/doc/ahess_03952649_1989_num_44_3_283612_t1_05 81 _0000_001

-http://www.lemonde.fr/culture/article/2009/05/30/jean-christophe-rufinune-vie-de-roman_1200289_3246.html

\section{DOCUMENTS SONORES}

-http://www.lesideesmenentlemonde.fr/821-jean-christophe-rufin.htm, entretien

avec Philippe Lapousterle

- https://www.youtube.com/watch?v=S_1XR_EL3oU, Entretien Le Point avec

Jean-Christophe Rufin

\section{RÉFÉRENCES EN ARABE}

كريستوفر مارلو، المذبحة فى باريب، نرجمة نوفيق على منصور ،تقديم فتحية الفرارجى، القاهرة،

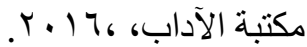

(MARLO Christopher, Le massacre à Paris, traduction de Tawfique Mansour présentation de Fathéya Al Fararguy, Le Caire, Librairie des Lettres, 2016.) 
